\title{
Heritability Estimates of Complex Intelligence and Associated Genetics
}

\author{
Nyomi Bourbous
}

\section{Abstract}

It has always aroused curiosity in the field of human science studies as to how much of ourselves can be genetically attributable to our parents. For relatively simple traits, like earwax consistency and albinism, this poses no problem as they can be followed genetically using simple Mendelian methods. When it comes to more complex constructs, however, such as cognitive capabilities, it is difficult to determine, to say the least. Intelligence is one facet of human cognition that inspires awe and curiosity among scientists. Whether a person is born with a particular intelligence measure (nature), or if intelligence is developed through a nurturing and stimulating environment (nurture) is an ongoing debate. Evidence exists for both sides of the argument, but the most compelling comes from the use of genetically identical individuals whose variance in intelligence can perhaps answer questions regarding the impact of one's surroundings on this complex cognitive capacity.

\section{Keywords}

Heritability. Intelligence. Genes. Environment. Cognitive Abilities.

\section{Introduction}

It has been shown that certain physical characteristics, such as finger lengths, correlate strongly with behaviours and personality (Paul et al., 2006). Thus it would make sense to assume that intelligence is also inherited. This assumption, however, underestimates the complexity of the nature of intelligence. When researching the heritability of intelligence, several factors come into play. Firstly, how can intelligence be quantified? What tests, definition, skills and traits make someone more intelligent than another? Is there a gene (or a set of genes) that localises intelligence to specific regions in the genome? Finally, it is imperative 
to discuss the nature versus nurture debate; are people born smart or do they become intelligent through upbringing and exposure to the right environment? The debate surrounding whether or not intelligence is inherited, much like physical characteristics, is nothing new. This essay shall attempt to answer all these questions as well as examine the validity of intelligence as a component of genetics and the environment.

\section{What is Intelligence?}

Over the centuries, the subjective nature of what constitutes intelligence has given rise to various theories in the philosophical and scientific communities. The two main models, which have been at the forefront and therefore heavily debated, are the theory of multiple intelligences (MI) and the theory of general cognitive ability, denoted by the factor ' $\mathrm{g}$ ' (Deary et al., 2009).

In 1986 Gardner expanded upon the Primary Mental Abilities Theory (formally established in 1938 by Thurstone) with the design of his own theory; that of Multiple Intelligence (Morgan, 1996). This theory stipulates that there are eight categories (not necessarily numerically based) for which one may display performance exceeding that of the general population, or in other words, the average. These include linguistic, logical-mathematical, musical, spatial, bodily kinaesthetic, intrapersonal, interpersonal and naturalistic intelligence (Traub, 1998). If an individual exceeds the ability of the general population in one of these fields of expertise, then that individual is deemed intelligent in that specific field. Gardner asserts that intelligence should not be determined by a single intellectual aptitude, but rather on a scope of independent variants (Traub, 1998).

In light of this account, Deary et al. (2009) claim that the MI theory holds no leverage in comparison with Charles Spearman's 1916 theory of general cognitive ability, which accounts for 40-50 per cent of intellectual variance. This model works by calculating a numerical value of mental aptitude, the general cognitive factor or $g$, using the technique of factor analysis across a variety of tests. Spearman concluded that individuals who performed well on one cognitive test generally did so across the remainder. Conversely, if a poor result was obtained this was also reflected in the others (Kuncel et al., 2004). Ultimately the g factor represents a general level of intelligence that is applicable to everyone. It is when one displays a greater intellectual capacity than this average that intelligence can be noted. Although there is some debate regarding the specific test variances giving rise to $g$, these are irrelevant as it is principally g that carries the predictive validity as well as variance of the intelligence trait (Deary et al., 2009). 
A variety of tests exist today that attempt to measure and quantify intelligence. Derived from the earlier Binet-Simon Scale, the Stanford-Binet Intelligence Quotient (IQ) Test founded in 1916 is considered to be one of the most valid tests. Debate continues with regard to its validity based upon cultural biases, gender differences and heritability. Most modern forms of the intelligence test have been specifically modified to remove as much of these as possible and instead compare individuals within their own age group (Bartholomew, 2004).

As is clear, there are a variety of approaches to understanding and measuring intelligence. Yet the underlying mechanisms that enable the existence of the enormous variety of intellectual traits are still unknown.

\section{The Heritability of Complex Traits}

In order to assess whether a genetic link exists between genes and intelligence, it is important to determine what genetic heritability is and whether it is a causal factor for assuming characteristics from one's parents. Heritability is recognised as the degree of phenotypic variation for a given trait resulting from genetic variation (Mackintosh, 1998). When it comes to simple Mendelian traits, such as earwax consistency and albinism, it is easy to distinguish whether genetics play a significant role in the phenotype of an individual as it simply requires the presence of a given gene. Yet cognitive abilities, like intelligence, are complex traits in which many genes accounting for small effects are hypothesised to contribute to their variation (Luciano et al., 2006). For this reason they are difficult to study. For example, to find a gene responsible for haemophilia, it is only necessary to look at a family history and extrapolate the genotypes from the phenotypes displayed throughout the pedigree. But with complex traits, multiple genes interact and are differentially expressed in individuals. Thus it is difficult to pinpoint where in the genome such genes exist.

\section{The Heritability of Intelligence}

For centuries people have contemplated whether a link exists between genes and intelligence. As technology and communication increase we are seeing more cases of siblings, such as the members of the British Imafidon family (Campbell, 2009), arise whereby multiple individuals within families exhibit superior intellectual capacities, which would not occur through random variation. Such examples lead to the question of whether intelligence has a genetic basis.

The development of the ability to map the human genome has enabled scientists to map specific genes that may relate to intelligence. The two main strategies 
in finding such genes include linkage analysis and candidate-gene association (Posthuma \& Geus, 2006). Linkage analysis is a method by which multiple markers affixed to known locations are correlated with the presence of certain phenotypes. If genes are found in close proximity to such markers then it makes it easier to locate them. This is because if the inheritance of a marker and trait can sufficiently be proven to occur together, 'then one or more genes in the vicinity of the marker are possibly involved in the trait similarity because genetic similarity on chromosomes is passed on in chunks' (Posthuma \& Geus, 2006). Conversely, candidate-gene association has been described as the process in which genes previously associated with a characteristic or a trait are tested to discern whether a relationship exists between them (Patnala et al., 2013). This is done by comparing the gene frequency of individuals who are known to express the phenotype against control subjects; i.e., individuals who do not express the trait. Association is inferred if the gene is observed at a significantly greater frequency in individuals expressing the trait (over control subjects).

It is important to note, however, that both these techniques are relatively new. While only a limited amount of research has been performed on their accuracy, studies were conducted, specifically in 2005 and 2006, that used linkage analysis on intelligence (Buyske et al., 2006; Dick et al., 2006; Luciano et al., 2006; Posthuma et al., 2005; Wainwright et al., 2006). The information gathered from these studies suggests that there are multiple genes, spanning 15 chromosomes (out of the 24, including $\mathrm{x}$ and $\mathrm{y}$ as separate units), that may contribute to intelligence (see Figure 1). More specifically it is unknown if and how these genes are related to intelligence even though many of them are found to be weakly correlated with the phenotypes that make up intelligence. Two regions on chromosomes 2 and 6 have, however, been positively identified as contributing to intelligence (Luciano et al. 2006; Posthuma et al., 2005).

Research using model-free multipoint linkage analysis has determined that region q24.1-31.1 of chromosome 2 and region p25.3-22.3 of chromosome 6 appear to display high correlations with a quantitative-trait locus for performance (perceptual organisation) intelligence quotient (IQ) and a suggestive linkage for both full-scale (cognitive capacity) and verbal (verbal comprehension) IQ respectively (Posthuma et al., 2005). These regions were identified because they presented a high correspondence between intellect and certain markers. The region of chromosome 2 highlighted an association with performance IQ (PIQ) and the marker D2S2330, while chromosome 6 indicated an association between full scale IQ (FSIQ) and verbal IQ (VIQ) via the marker D6S942.

Even more interesting is that each of these regions has been connected with various mental disorders (Posthuma et al., 2005). The q24.1-31.1 region of chromosome 2 largely overlaps with the 2q21-33 region, which has been strongly linked to autism by at least four independent genetic studies (Philippe et al., 
1999; Buxbaum et al., 2001; Palferman et al., 2001; Shao et al., 2002). Likewise, the p25.3-22.3 region of chromosome 6 also displays an overlapping region that is related to reading disabilities and dyslexia. Most intriguingly, several genes exist within these linkage regions that have been associated with schizophrenia, fragile-X syndrome and Bardet-Biedl syndrome - disorders that are typically accompanied by cognitive impairments.

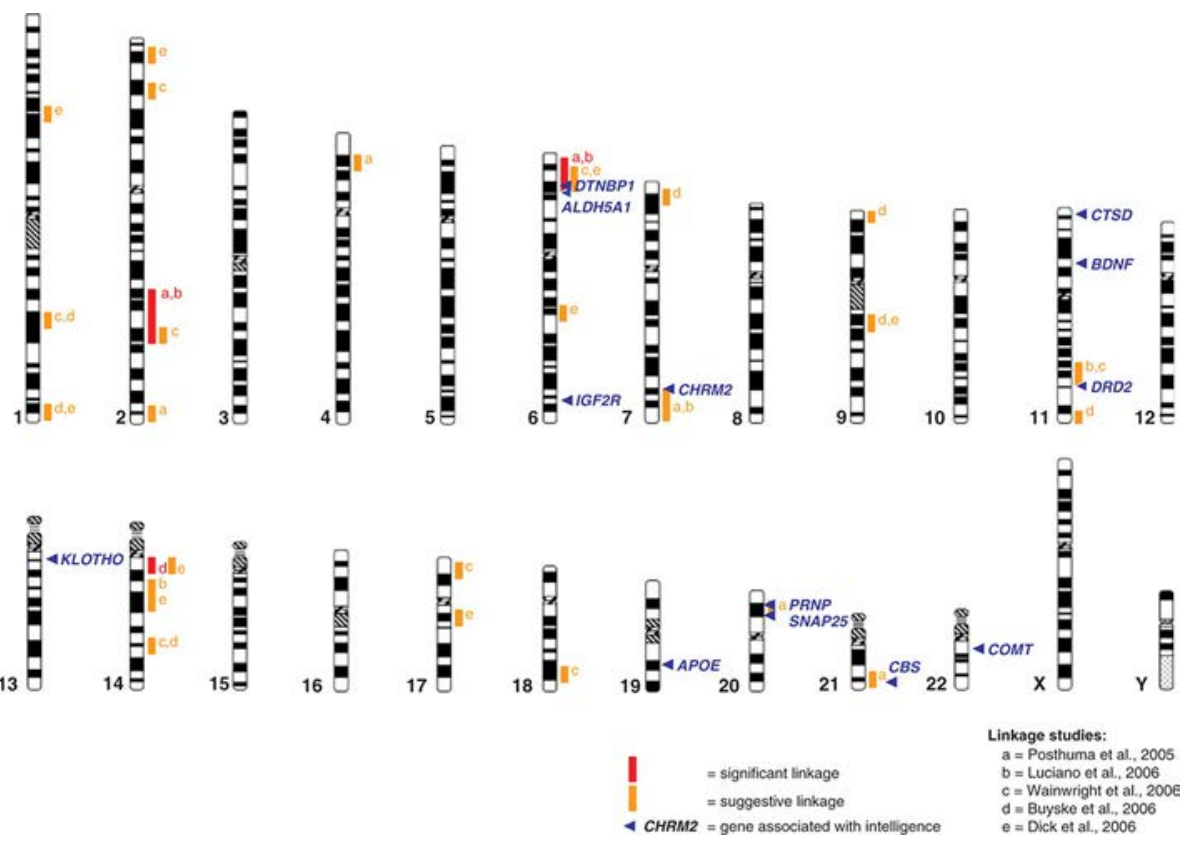

Figure 1: An ideogram of the human genome (based on the five linkage studies of intelligence conducted to date) highlighting chromosomal regions (denoted by orange) containing genes already associated with intelligence and regions (denoted by red) believed to contain genes associated with intelligence.

Source: Posthuma \& Geus, 2006.

A 2006 study (Luciano et al.) reported similar results to those described above (Posthuma et al., 2005). The study found that there are regions in chromosomes 2 and 6 that correlate with PIQ and FSIQ/VIQ respectively. It also demonstrated that the linkage region of chromosome 2 not only correlated for PIQ, but also for word recognition. This confirmed that there could be individual genes that influence different aspects of cognitive ability (Plomin \& Kovas, 2005).

Genetic analysis provides an understanding of the correlations between genes and phenotypes through testing IQ scores and comparing them to genes. This does not, however, test for the interactions between nature and nurture. Indeed, 
epigenetics further complicates the connection between intelligence and genetic heritability. Twin studies have been used in attempts to separate the effect of the environment from that of the genes.

\section{The Relevance of Twin Studies and Adoption Studies}

Twin studies and adoption studies have been undertaken since the late nineteenth century, when they were first utilised by Sir Francis Galton (Yong-Kyu, 2009). Galton surmised that the more genetically alike two individuals are, the more similar their physical and mental characteristics.

Monozygotic twins share 100 per cent of their genes while fraternal twins are exactly like individually born siblings and share approximately 50 per cent of their genes. It logically follows then that 'if identical twins are more similar than fraternal twins on a particular outcome, then genetic influences are assumed' (Wilhelm \& Engle, 2005). Likewise, if adopted children are more similar to their adoptive parents and siblings, then environmental influences are implicated. Twin and adoptive studies are thus frequently used in genetic research as means of ascertaining environmental and genetic influences on different traits.

Such studies have advanced genetic research. Unlike rare, clinical conditions, which can be diagnosed by observing a regular pattern of inheritance throughout a family's history, complex traits cannot be measured in this way (Johnson et al., 2009). As discussed, there are multiple genes that interact to magnify or reduce the effect of other genes. Twin studies allow researchers to effectively separate the gene's effects from the environment. Monozygotic twins reared apart are genetically identical individuals living in different environments. By examining the differences that exist between them in particular characteristics (e.g., IQ scores), it is possible to ascertain the effect of the environment. The downside to these studies is that they require large sample sizes from monozygotic twin populations (which are rare) in order to accumulate sufficient statistical power (Johnson et al., 2009).

\section{The Nature versus Nurture Debate}

At the forefront of human genetic research exists the debate as to whether intelligence is a preconceived trait or one acquired over an individual's lifetime. Although when first established in the 1970s the debate consisted of two clear unambiguous positions, that of nature versus nurture, more recently it 
has moved on to the theme of nature via nurture (Anderson, 1999). Nature via nurture means that both components contribute differently to intelligence and that, symbiotically, one cannot work without the other.

Today there is no denying that the heritability of intelligence is one of the most consistent findings in the scientific literature (Wilhelm \& Engle, 2005), which has been determined mainly by twin and adoption studies (Deary et al., 2009). These methods have found significant correlations between age and pronounced intellectual heritability as well as socio-economic status and intelligence. Unlike Mendelian genetics, intelligence does not exhibit a uniform hereditary status throughout one's lifetime. Instead, with the assistance of twin studies, it has been found that generally, heritability of intelligence increases from around 20 per cent in infancy to 60 per cent in adulthood onward to 80 per cent in old age (Wilhelm \& Engle, 2005). This information reveals that nurture is significant to the growth and developmental expression of our genes. Similarly, analysis of the heritability of intelligence as impacted by socio-economic upbringing has found that individuals raised in low socio-economic environments display low heritability of intelligence while individuals raised in high socio-economic environments display increased levels of heritability (Posthuma \& Geus, 2006). This also indicates that nurture, via our environment, is influential in the expression of intelligence.

Ultimately with the assistance of twin studies and new genetic technology the nurture-nature debate is leaning in the direction that both components are significant to the development of intelligence. The naturalistic components are the genetic contributors and it is acknowledged that genes form the basis upon which intelligence can be established and that everybody possesses a basic intellectual capacity (denoted by the value g). Also, the component of nurture is required to further enhance an individual's intelligence, which involves the development of neurological pathways and thought processes. Yet it is the maturation of these cognitive processes that sees them switch from being environmentally activated to having a genetic basis.

It can be concluded that, although nature is a necessary component to providing a foundation upon which intelligence can grow, nurture is just as crucial to continue building upon this foundation, for it appears that an environment void of intellectual stimuli would inhibit this growth. 


\section{Possible Future Directions for the Study of Intelligence}

The field of genetic inheritance of intelligence is recently established, mainly because the mapping of the human genome concluded only fairly recently, especially when compared to the field of intelligence and its heritability as a whole. As this essay has demonstrated, new research has deduced that intelligence does indeed have a genetic basis, with specific areas in chromosomes 2 and 6 possibly being influential. Although this is the case, researchers are still unable to infer how these areas contribute. Future investigation of this relationship will assist the medical world to understand the development of intelligence and the biological meaning of its apparently high heritability (Deary et al., 2009). Additionally, it would open new windows into the investigation of mental disorders and their diagnosis, as well as possible treatments and cures.

With regard to the influence of nurture on the expression of intelligence, it has been established that everybody possesses a basic level of intelligence. Heritability is, however, increasingly expressed with age and as a result of an advantageous socio-economic upbringing. This suggests that the greater the development of the individual's brain, whether as a result of growth or external stimuli, the more intelligence functions will begin to resemble those of the parents.

Another promising route for future research into intelligence is the study of knockout genes. This method attempts to ascertain whether an individual exhibits a faulty gene (or lack of it) and whether its absence impairs their intellectual aptitude. Genetic knockout experiments on humans would give rise to many ethical objections, which may be overcome by studying individuals who already exhibit these mutations and by conducting animal model experiments.

In conclusion, it is clear that genes and an individual's environment influence intelligence. To place an exact number or percentage on this influence would, however, be nearly impossible as there are multiple factors that determine the proportional influence of each.

\section{Bibliography}

Anderson M (1999), The Development of Intelligence, Psychology Press, England. Bartholomew D (2004), Measuring Intelligence: Facts and Fallacies, Cambridge University Press, United Kingdom. 
Buxbaum JD, Silverman JM, Smith CJ, Kilfarski M, Reichert J (2001), 'Evidence for a Susceptibility Gene for Autism on Chromosome 2 and for Genetic Heterogeneity', The American Journal of Human Genetics, Volume 68, Issue 6, pp. 1514-520.

Buyske S, Bates ME, Gharani N, Matise TC, Tishfield JA (2006), 'Cognitive Traits Link to Human Chromosomal Regions', Behavioral Genetics, Volume 36, Issue 1 , pp. 65-76.

Campbell D (2009), 'Brainy Twins Smash Math Record', Sky News Online, <http://news.sky.com/home/uk-news/article/15204296>.

Collins F (1999), 'Medical and Societal Consequences of the Human Genome Project', The New England Journal of Medicine, Volume 341, Issue 1, pp 28-37.

Deary IJ, Johnson W, Houlihan LM (2009), 'Genetic Foundations of Human Intelligence', Human Genetics, Volume 126, Issue 1, pp. 215-32.

Dick DM, Aliev F, Bierut L, Goate A, Rice J (2006), 'Linkage Analyses of IQ in the Collaborative Study on the Genetics of Alcoholism (COGA) Sample', Behavior Genetics, Volume 36, Issue 1, pp. 77-86.

Johnson W, Turkheimer E, Gottesman II, Bouchard TJ (2009), 'Beyond Heritability Twin Studies in Behavioral Research', Current Directions in Psychological Science, Volume 18, Issue 4, pp. 217-20.

Kuncel NR, Hazlett SA, Ones DS (2004), 'Academic Performance, Career Potential, Creativity, and Job Performance: Can One Construct Predict Them All?', Journal of Personality and Social Psychology, Volume 86, Issue 1, pp. 148.

Luciano M, Wright MJ, Duffy DL, Wainwright MA, Zhu, G (2006), ‘Genomewide Scan of IQ Finds Significant Linkage to a Quantitative Trait Locus on $2 q^{\prime}$, Behavior Genetics, Volume 36, Issue 1, pp. 45-55.

Mackintosh N (1998), IQ and Human Intelligence, Oxford University Press, England.

Morgan H (1996), 'An Analysis of Gardner's Theory of Multiple Intelligence', Roeper Review, Volume 18, Issue 4, pp. 263-69.

Palferman S, Matthews N, Turner M, Moore J, Hervas A (2001), ‘A Genome Wide Screen for ASutism: Strong Evidence for Linkage to Chromosomes 2q, 7q, and 16p', American Journal of Human Genetics, Volume 69, Issue 3, pp. 570-81. 
The ANU Undergraduate Research Journal

Patnala R, Clements J, Jyotsna B (2013), 'Candidate Gene Association Studies: A Comprehensive Guide to Useful in Silico Tools', BMC Genetics, Volume 14, Issue 1, pp. 39.

Paul SN, Kato BS, Cherkas LF, Andrew T, Spector TD (2006), 'Heritability of the Second to Fourth Digit Ration (2d:4d): A Twin Study', Twin Research and Human Genetics, Volume 9, Issue 2, pp. 215-19.

Philippe A, Martinez M, Guilloud-Bataille M, Gillberg C, Rastam M (1999), 'Genome-wide Scan for Autism Susceptibility Genes', Hum Molecular Genetics Genes, Volume 8, Issue 5, pp. 805-12.

Plomin R, Kovas Y (2005), 'Generalist Genes and Learning Disabilities', Psychological Bulletin, Volume 131, Issue 4, pp. 592.

Posthuma D, Luciano M, Geus EJD, Wright MJ, Slagboom PE (2005), 'A Genomewide Scan for Intelligence Identifies Quantitative Trait Loci on $2 \mathrm{q}$ and 6', American Journal of Human Genetics, Volume 77, Issue 2, pp. 318-26.

Posthuma D, Geus EJD (2006), 'Progress in the Molecular Genetic Study of Intelligence', Current Directions in Psychological Science, Volume 15, Issue 4, pp. 151-55.

Shao Y, Raiford KL, Wolpert CM, Cope HA, Ravan SA (2002), 'Phenotypic Homogeneity Provides Increased Support for Linkage on Chromosome 2 in Autistic Disorder', American Journal of Human Genetics, Volume 70, Issue 4, pp. 1058-61.

Traub J (1998), 'Multiple Intelligence Disorder', The New Republic, Volume 1, p. 20.

Thurstone L.L. (1938), Primary Mental Abilities, (Psychometric Monographs, Volume 1), University of Chicago Press, Chicago, United States of America.

Wainwright MA, Wright MJ, Luciano M, Montgomery GW, Geffen GM, (2006), 'A Linkage Study of Academic Skills Defined by the Queensland Core Skills Test', Behavioral Genetics, Volume 36, Issue 1, pp. 56-64.

Wilhelm O, Engle RW (2005), Handbook of Understanding and Measuring Intelligence, Sage Publications, United States of America.

Yong-Kyu K (2009), Handbook of Behavior Genetics, Springer Publisher, New York, United States of America. 
This text taken from The ANU Undergraduate Research Journal Volume Five 2013, published 2014 by ANU Press, The Australian National University, Canberra, Australia. 